\section{Methemoglobinemia in Patient with G6PD Deficiency and SARS-CoV-2 Infection}

\author{
Kieran Palmer, Jonathan Dick, Winifred French, \\ Lajos Floro, Martin Ford
}

Author affiliation: King's College Hospital National Health Service Foundation Trust, London, UK

DOI: https://doi.org/10.3201/eid2609.202353

We report a case of intravascular hemolysis and methemoglobinemia, precipitated by severe acute respiratory syndrome coronavirus 2 infection, in a patient with undiagnosed glucose-6-phosphate dehydrogenase deficiency. Clinicians should be aware of this complication of coronavirus disease as a cause of error in pulse oximetry and a potential risk for drug-induced hemolysis.

Coronavirus disease is a novel infectious disease that primarily manifests as an acute respiratory syndrome but can also cause multiorgan dysfunction. Severe acute respiratory syndrome coronavirus 2 (SARS-CoV-2) infection has been documented to cause vasoocclusive crisis and acute chest syndrome in patients with sickle cell anemia (1). We report another potentially major complication of infection in a patient with a common enzymatic disorder.

Glucose 6-phosphate dehydrogenase (G6DP) deficiency is an X-linked enzymatic disorder that affects 400 million persons worldwide and has a high prevalence $(5 \%-20 \%)$ in African and Asian populations (2). G6DP catalyzes the formation of nicotinamide adenine dinucleotide phosphate (NADPH) (3). NADPH maintains hemoglobin in the ferrous state by forming reduced glutathione, which prevents oxidative damage (3). G6DP deficiency increases the risk for intravascular hemolysis upon exposure to oxidative agents, such as fava beans, sulfonamides, and hydroxychloroquine, the subject of clinical trials for persons with SARS-CoV-2 infection.

G6PD deficiency can induce methemoglobinemia by inhibiting NADPH-flavine reductase, which prevents the reduction of methemoglobin. Methemoglobin is unable to bind to oxygen, and the remaining oxyhemoglobin develops heightened oxygen affinity and diminished delivery, leading to tissue hypoxia (4). Viral infections, including HIV, hepatitis viruses (A, $\mathrm{B}$, and $\mathrm{E})$, and cytomegalovirus, can precipitate intravascular hemolysis in patients with G6PD deficiency $(5,6)$. Concurrent methemoglobinemia has also been reported in the context of viral-induced hemolysis (5).
A 62-year-old Afro-Caribbean man with a medical history of type 2 diabetes and hypertension came to the hospital for a 5-day history of fever, dyspnea, vomiting, and diarrhea. Auscultation of his chest showed bilateral crackles. He was tachycardic, hypotensive, and dehydrated, with a prolonged capillary refill time and dry mucous membranes.

Laboratory tests showed an acute kidney injury. Blood urea nitrogen was $140 \mathrm{mg} / \mathrm{dL}$, creatinine 5.9 $\mathrm{mg} / \mathrm{dL}$ (baseline $1.1 \mathrm{mg} / \mathrm{dL}$ ), capillary blood glucose $>31 \mathrm{mmol} / \mathrm{L}$, and blood ketones $1.1 \mathrm{mmol} / \mathrm{L}$. A chest radiograph showed bilateral infiltrates, and a result for a SARS-CoV-2 reverse transcription PCR specific for the RNA-dependent RNA polymerase gene was positive (validated by Public Health England, London, UK).

The patient was treated for SARS-CoV-2 pneumonitis and a hyperosmolar hyperglycemic state with crystalloid fluid, oxygen therapy, and an insulin infusion. His creatinine increased to $9.3 \mathrm{mg} / \mathrm{dL}$, suspected secondary to hypovolemia and viremia, and acute hemodialysis was started. Results of a screen for other causes of acute kidney injury, including renal ultrasonography and autoimmune serologic analysis, was unremarkable.

On day 7 postadmission, his peripheral oxygen saturations decreased, and oxygen therapy was increased to $15 \mathrm{~L} / \mathrm{min}$ by use of a nonrebreather mask to maintain saturations of $80 \%$. Arterial blood gas analysis revealed a partial pressure of oxygen of $22 \mathrm{kPa}$ and an oxygen saturation of $100 \%$. Co-oximetry showed a methemoglobin level of $6.5 \%$. Repeat laboratory tests showed hemolytic anemia; hemoglobin was $52 \mathrm{~g} / \mathrm{L}$, haptoglobin $<0.1 \mathrm{mg} / \mathrm{dL}$, and lactate dehydrogenase 1,566 U/L. A direct antiglobulin test excluded major immune-mediated hemolysis. A blood film for the patient showed normochromic normocytic erythrocytes and a few hemighost cells (Figure, panel A). A 2-stage G6DP assay confirmed G6DP deficiency (0.8 $\mathrm{IU} / \mathrm{g}$ hemoglobin).

The patient was given 2 blood transfusions (Figure, panel B) and oxygen therapy. His medication history included amoxicillin/clavulanic acid, heparin, amlodipine, and metformin, which did not indicate a precipitant for the hemolytic crisis. The methemoglobinemia gradually resolved, and his oxygen requirements decreased. He recovered dialysis-independent renal function. He was given folic acid $(5 \mathrm{mg} / \mathrm{d})$ and discharged 22 days after admission.

The mechanism by which SARS-CoV-2 causes hemolysis is unknown. Other viral infections have been reported to produce reactive oxygen and nitrogen species, which impair intracellular proteins and DNA 

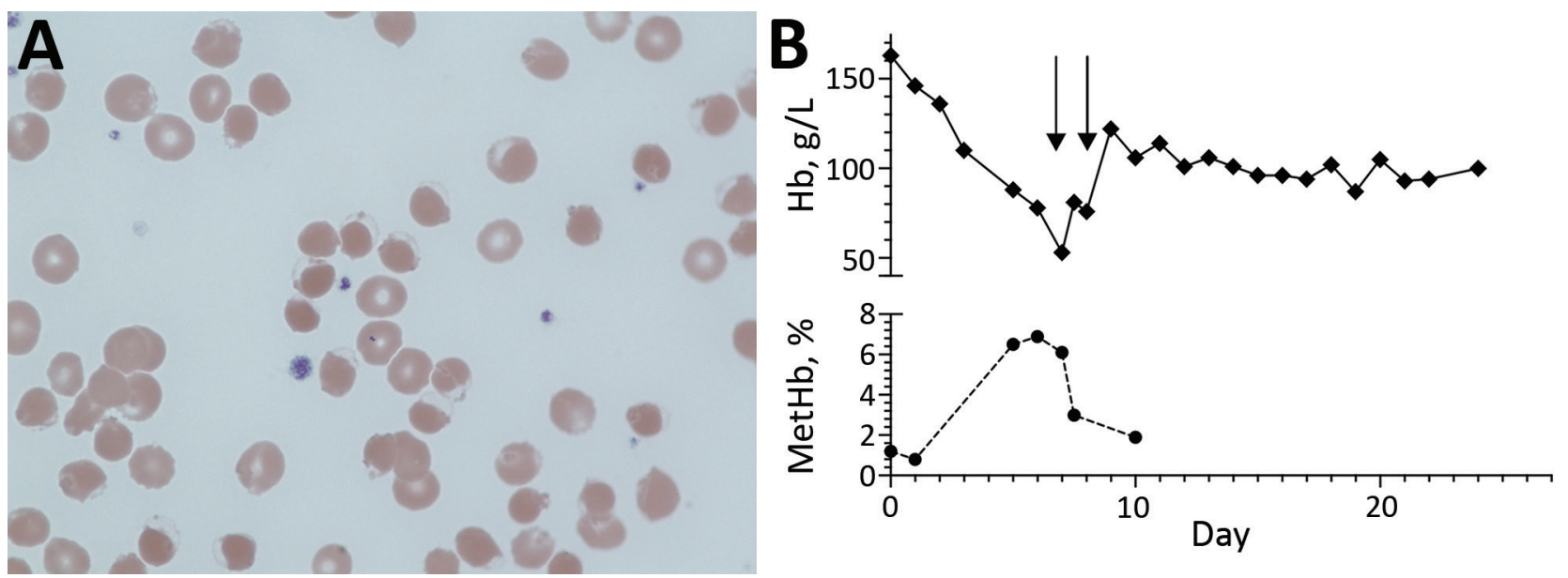

Figure. Testing of patient with G6PD deficiency and SARS-CoV-2 infection, United Kingdom. A) Blood film showing normochromic normocytic erythrocytes and a few hemighost cells. Hemighost cells are formed after oxidative hemolysis seen in G6DP deficiency. Hb is contracted to 1 pole of the cell, leaving an unfilled area enclosed by an intact membrane (original magnification $\times 100$ ). B) $\mathrm{Hb}$ and metHb concentration during admission. Each arrow indicates a 3-unit erythrocyte transfusion. G6PD, glucose-6-phosphate dehydrogenase; Hb, hemoglobin; metHb, methemoglobin; SARS-CoV-2, severe acute respiratory syndrome coronavirus 2.

in cells with damaged antioxidant enzyme metabolism (7). The concurrent secondary methemoglobinemia in this case also suggests oxidative stress and impaired redox balance.

Patients with G6DP deficiency might be more vulnerable to SARS-CoV-2 infection (8). Infection of G6DP-deficient lung cells with human coronavirus $229 \mathrm{E}$ resulted in increased viral production and replication compared with normal cells (9). An increased susceptibility to infection and hemolysis with secondary tissue hypoxia might result in increased illness and death (8).

Hydroxychloroquine has been proposed as a treatment for SARS-CoV-2 infection and is considered safe in usual therapeutic doses in class II or III G6PD deficiency. However, caution is advised with higher doses because data for this setting are limited. Oxidative stress might contribute to the pathogenesis of severe SARS-CoV-2 infection (10). Evaluation of parameters of oxidative stress in SARS-CoV-2 are currently underway (ClinicalTrials.gov identifier NCT04375137) and might determine whether there is an increased risk for drug-induced hemolysis in patients with G6PD deficiency.

Treatment for methemoglobinemia with intravenous methylene blue is recommended if the blood methemoglobin level is $>20 \%-30 \%$. However, in G6DP deficiency, treatment with methylene blue is contraindicated because the reduction of methemoglobin is NADPH dependent. This finding might precipitate intravascular hemolysis and therapy with ascorbic acid or supportive treatment with oxygen as indicated instead. During the SARS-CoV-2 pandemic, clinicians must be aware of the possible increased susceptibility of patients with G6DP deficiency to severe hemolytic crises and the consequences for investigation and treatment.

\section{About the Author}

Dr. Palmer is an internal medicine trainee in the Renal Department, King's College Hospital NHS Foundation Trust, London, UK. His primary research interests are infectious diseases and general medicine.

\section{References}

1. Nur E, Gaartman AE, van Tuijn CF, Tang MW, Biemond BJ. Vaso-occlusive crisis and acute chest syndrome in sickle cell disease due to 2019 novel coronavirus disease (COVID-19). Am J Hematol. 2020;95:725-6. https:/ / doi.org/10.1002/ ajh.25821

2. Cappellini MD, Fiorelli G. Glucose-6-phosphate dehydrogenase deficiency. Lancet. 2008;371:64-74. https://doi.org/10.1016/S0140-6736(08)60073-2

3. Efferth T, Schwarzl SM, Smith J, Osieka R. Role of glucose-6-phosphate dehydrogenase for oxidative stress and apoptosis. Cell Death Differ. 2006;13:527-8, author reply 529-30. https:/ / doi.org/10.1038/sj.cdd.4401807

4. Hassan KS, Al-Riyami AZ, Al-Huneini M, Al-Farsi K, Al-Khabori M. Methemoglobinemia in an elderly patient with glucose-6-phosphate dehydrogenase deficiency: a case report. Oman Med J. 2014;29:135-7. https://doi.org/ 10.5001/omj.2014.33

5. Au WY, Ngai CW, Chan WM, Leung RY, Chan SC. Hemolysis and methemoglobinemia due to hepatitis E virus infection in patient with G6PD deficiency. Ann Hematol. 2011;90:1237-8. https://doi.org/10.1007/s00277-011-1167-6

6. Araujo T, Katiyar V, Gonzales Zamora JA. Acute retroviral syndrome presenting with hemolytic anemia induced by G6PD deficiency. Trop Med Infect Dis. 2018;4:6. https://doi.org/10.3390/tropicalmed4010006 
7. Aydemir D, Ulusu NN. Is glucose-6-phosphate dehy (COVID-19) infections and deaths? Pathog Glob Health. 2020; 114:109-10. https:/ / doi.org/10.1080/20477724.2020.1751388

8. Kassi EN, Papavassiliou KA, Papavassiliou AG. G6PD and chloroquine: selecting the treatment against SARS-CoV-2? J Cell Mol Med. 2020;24:4913-4. https:/ / doi.org/10.1111/ jcmm.15312

9. Wu YH, Tseng CP, Cheng ML, Ho HY, Shih SR, Chiu DT. Glucose-6-phosphate dehydrogenase deficiency enhances human coronavirus 229E infection. J Infect Dis. 2008; 197:812-6. https://doi.org/10.1086/528377

10. Delgado-Roche L, Mesta F. Oxidative stress as key player in severe acute respiratory syndrome coronavirus (SARS-CoV) infection. Arch Med Res. 2020. Apr 30 [Epub ahead of print]. https://doi.org/10/1016/j.arcmed.2020.04.019.

Address for correspondence: Kieran Palmer, Renal Department, King's College Hospital National Health Service Foundation Trust, Denmark Hill, Brixton, London SE59RS, UK; email: kieran. palmer3@nhs.net

\section{Asymptomatic SARS-CoV-2 Infection in Nursing Homes, Barcelona, Spain, April 2020}

\author{
Blanca Borras-Bermejo, ${ }^{1}$ Xavier Martínez-Gómez, ${ }^{1}$ \\ María Gutierrez-San Miguel, Juliana Esperalba, \\ Andrés Antón, Elisabet Martin, Marta Selvi, \\ María José Abadías, Antonio Román, \\ Tomàs Pumarola, Magda Campins, Benito Almirante \\ Author affiliations: Vall d'Hebron Hospital Universitari, Barcelona, \\ Spain (B. Borras-Bermejo, X. Martínez-Gómez, M. Gutierrez-San \\ Miguel, J. Esperalba, A. Antón, M.J. Abadías, A. Román, \\ T. Pumarola, M. Campins, B. Almirante); Universitat Autònoma de \\ Barcelona, Bellaterra, Spain (X. Martínez-Gómez, A. Antón, \\ A. Román, T. Pumarola, M. Campins, B. Almirante); Servei Atenció \\ Primària Muntanya, Barcelona (E. Martin); Centre d'Atenció Primària \\ Sant Andreu, Barcelona (M. Selvi)
}

DOI: https://doi.org/10.3201/eid2609.202603

During the coronavirus disease pandemic in Spain, from April 10-24, 2020, a total of 5,869 persons were screened for severe acute respiratory syndrome coronavirus 2 at nursing homes. Among residents, 768 (23.9\%) tested positive; among staff, $403(15.2 \%)$. Of those testing positive, $69.7 \%$ of residents and $55.8 \%$ of staff were asymptomatic. s of April 2020, Spain was one of the countries accounting for the most coronavirus disease (COVID-19) deaths (1). More than half of those deaths occur in persons $>80$ years of age (2), which highlights the vulnerability of the elderly. Moreover, severe acute respiratory syndrome coronavirus 2 (SARS-CoV-2) can be easily spread within nursing homes, causing outbreaks with high associated mortality rate $(3,4)$. By the beginning of April, the exponential increase of cases overwhelmed the healthcare system in Spain. In this context, rapid outbreak identification and early intervention in nursing homes was needed.

At Vall d'Hebron Hospital, a tertiary hospital in Catalonia, Spain, we conducted test-based screening as a containment measure to promptly implement effective prevention and control measures in nursing homes. We present the early results of a coordinated intervention with primary care teams in $\approx 6,000$ residents and facility staff in nursing homes in our catchment area.

We evaluated 69 nursing homes that had a total census of 6,714 persons. We excluded previous laboratory-confirmed cases of COVID-19. During April 10-24, an integrated team of hospital and primary care staff obtained samples for SARS-CoV-2 testing from all residents and workers: nasopharyngeal and oropharyngeal swab samples both combined in the same collection tube with viral transport media. We used a commercial CE-IVD-marked, real-time reverse transcription PCR-based assay (Cobas SARSCoV-2; Roche Diagnostics, https:/ / www.roche.com) on a Cobas 6800 system.

Each nursing home director recorded any symptoms present at least 48 hours before the scheduled day of testing for all residents and staff. According to the World Health Organization case definition of a suspected case of COVID-19, a person was classified as symptomatic if fever or acute respiratory symptoms were present at any moment during the preceding 14 days. In the absence of either, the person was considered to be asymptomatic.

We obtained a total of 5,869 samples, 3,214 from residents and 2,655 from facility staff. Overall, 768 $(23.9 \%)$ residents and $403(15.2 \%)$ staff members tested positive for SARS-CoV-2 (Table). The presence of fever or respiratory symptoms during the preceding 14 days was recorded in 2,624 residents (81.6\%) and 1,772 staff members $(66.7 \%)$. Among those testing positive and for whom we had information about symptoms, $69.7 \%$ of the residents and $55.8 \%$ of staff were asymptomatic.

${ }^{1}$ These first authors contributed equally to this article. 